\title{
ALTERAÇÕES ESTRUTURAIS DO SISTEMA RADICULAR DE SOJA EM RESPOSTA À DISPONIBILIDADE DE FÓSFORO NO SOLO ${ }^{1}$
}

\author{
Dioger Alexandre Teruel2,5*; Durval Dourado-Neto ${ }^{2,6}$; Jan W. Hopmans ${ }^{3}$; Klaus Reichardt",6 \\ ${ }^{2}$ Depto. de Produção Vegetal - USP/ESALQ, C.P. 9 - CEP: 13418-900 - Piracicaba, SP. \\ ${ }^{3}$ Dept. of Land, Air and Water Resources - University of California, Davis, USA. \\ ${ }_{5}^{4}$ Depto. de Ciências Exatas - USP/ESALQ. \\ ${ }^{5}$ Bolsista FAPESP. \\ ${ }^{6}$ Bolsista CNPq. \\ *Autor correspondente <teruel@sum.desktop.com.br>
}

\begin{abstract}
RESUMO: Tendo em vista que os métodos estatísticos tradicionais de análise dos atributos métricos do sistema radicular são insatisfatórios no que diz respeito à identificação e interpretação de estratégias adotadas pela planta em adaptação ao ambiente edáfico, o objetivo do trabalho foi utilizar conceitos de topologia para analisar as alterações estruturais apresentadas pelo sistema radicular da soja (Glycine max L. Merr. Cv. Williams 82) em resposta à disponibilidade de fósforo no solo. Plantas de soja foram cultivadas em recipientes de acrílico preto, em condições controladas de temperatura e fotoperíodo. Dois tratamentos foram estabelecidos, pela adição de solução nutritiva ao substrato dos recipientes: $+P\left(15,5 \mathrm{mg} \mathrm{L}^{-1} \mathrm{P}\right)$ e $-\mathrm{P}\left(8,5 \mathrm{mg} \mathrm{L}^{-1} \mathrm{P}\right)$. Utilizando-se delineamento experimental inteiramente casualizado, com parcelas subdivididas no tempo, três repetições foram amostradas aos 5, 10, 15, 20, 25 e 30 dias após a germinação. Para cada tratamento, idade de amostragem e repetição foram obtidos o comprimento da raiz principal (ordem 0), e os respectivos números e comprimentos médios das raízes laterais de ordens 1, 2 e 3, de acordo com a posição de origem na raiz principal. Índices topológicos foram definidos e aplicados para cada tratamento, utilizando-se os valores médios das três repetições. Os índices utilizados foram eficientes em detectar as alterações estruturais apresentadas pelos sistemas radiculares das plantas cultivadas sob menor teor de fósforo no solo.
\end{abstract}

Palavras-chave: Glycine max, topologia radicular, fósforo, nutrientes, estratégias adaptativas

\section{STRUCTURAL CHANGES IN SOYBEAN ROOT SYSTEMS AS A RESPONSE TO SOIL PHOSPHORUS AVAILABILITY}

\begin{abstract}
Since the analytical framework and quantitative tools associated with classical statistics are not efficient to identify and interpret plant strategies for adaptation to a given soil environment, the objective of this study was to utilize concepts of topology in order to analyze the structural changes in soybean root systems in response to soil phosphorus availability. Soybean plants were grown in black plexiglas boxes under controlled environment (temperature and photoperiod). The boxes were packed with substrate mixed with nutrient solution to achieve uniform $P$ concentration for two treatments: $+P\left(15.5 \mathrm{mg} \mathrm{L}^{-1} \mathrm{P}\right)$ and $-\mathrm{P}\left(8.5 \mathrm{mg} \mathrm{L}^{-1} \mathrm{P}\right)$. In a completely randomized experimental design, three replicates were sampled at $5,10,15,20,25$, and 30 days after germination. For each treatment, plant age, and replicate, the following attributes were obtained: main root length (order 0 ), and average number and length of order-1, order-2, and order-3 lateral roots according to their position of origin along the main root. Topological indexes were defined and calculated for both treatments, using the mean values of three replicates. Indexes used in this study were efficient in detecting the structural changes performed by the soybean root systems growing under lower soil P concentration.
\end{abstract}

Key words: Glycine max, root topology, phosphorus, soil nutrients, adaptive strategies

\section{INTRODUÇÃO}

Embora alguns nutrientes, como os nitratos, se movimentem com relativa liberdade em solos úmidos, tornando pouco importante a proximidade das superfícies radiculares de absorção à fonte do recurso, para nutrientes com baixas taxas de difusão no solo, como os fosfatos, a proximidade entre a superfície absortiva da raiz e a fonte do recurso é muito importante (Harper et al., 1991).
Neste último caso, o sistema radicular precisa explorar o solo em busca do recurso imóvel, e fazer uso do mesmo de maneira otimizada. Segundo Harper et al. (1991), o crescimento em extensão e a ramificação profusa provavelmente representam estratégias alternativas na exploração de volumes de solo e na aquisição dos recursos encontrados pelos sistemas radiculares, com diferentes conseqüências. Um sistema radicular pouco ramificado, com grande crescimento em extensão, não consegue fazer uso otimizado do recurso encontrado, enquanto que sistemas 
radiculares muito ramificados não podem descobrir novos recursos, pois tal estratégia adaptativa geralmente envolve menor crescimento em extensão, para que se mantenha praticamente inalterada a quantidade de fotoassimilados destinada ao sistema radicular. Tiffney \& Niklas (1985) afirmam que os sistemas radiculares podem, portanto, alterar sua configuração geométrica de forma a possuir a habilidade para explorar o solo em busca de recursos (grande crescimento em extensão, às custas de menor ramificação), ou a habilidade de adquirir os recursos encontrados de maneira eficiente (sistema radicular profusamente ramificado, às custas do crescimento em extensão).

Devido à extrema complexidade da estrutura geométrica de um sistema radicular, aliada à sua plasticidade (habilidade de mudar sua configuração em reação ao ambiente físico, químico e biológico do solo), as ferramentas analíticas e quantitativas para caracterização e interpretação deste sistema são ainda insatisfatórias (Robinson, 1996; Lynch, 1995), fazendo com que novas idéias e abordagens criativas sejam mais importantes que avanços tecnológicos que permitam a obtenção mais detalhada de atributos do sistema radicular (Robinson, 1991).

Uma metodologia de análise promissora para a quantificação e interpretação de sistemas radiculares é a análise baseada na topologia radicular (Fitter, 1985, 1987; Fitter et al., 1991). A topologia radicular se refere à maneira na qual segmentos gerados por processos de ramificação estão conectados uns aos outros dentro do sistema radicular. Ao contrário da arquitetura radicular, que se refere ao posicionamento no espaço tridimensional de cada segmento radicular individual, a topologia é estável à deformação ou rotação dos segmentos e pode, portanto, ser medida em sistemas radiculares retirados do solo por escavação (Lynch, 1995).

Pode-se encontrar dois extremos topológicos, um sendo o sistema radicular "espinha-de-peixe", que compreende um eixo primário individual e raízes laterais emergindo deste, e o outro o sistema radicular dicótomo, raramente encontrado em situações reais, no qual cada segmento radicular gera dois novos pela ramificação de seu ápice (Fitter et al., 1991).

A maioria dos trabalhos de pesquisa a respeito da estrutura e funcionamento de sistemas radiculares de espécies comerciais tem sido feita somente num pequeno número de espécies, principalmente monocotiledôneas (McCully, 1995). Com exceção de um grupo de pesquisadores trabalhando com Phaseolus vulgaris L. (Lynch \& Beebe, 1995; Lynch et al., 1997), são poucos os estudos conduzidos com dicotiledôneas herbáceas, sendo por isso utilizada a soja (Glycine max (L.) Merrill) neste estudo, dada a importância da cultura no Brasil e no mundo.

O objetivo do trabalho foi estudar as variações topológicas do sistema radicular de soja em resposta à disponibilidade de fósforo no solo, de maneira a identificar estratégias adaptativas utilizadas pela planta frente a mudanças em seu ambiente edáfico.

\section{MATERIAL E MÉTODOS}

\section{Procedimento experimental}

Neste estudo, realizado em condições de ambiente controlado nas dependências da Universidade da Califórnia em Davis, CA, EUA, plantas de soja (Glycine max L. Merrill cv. Williams 82) foram cultivadas em recipientes de acrílico preto com dois compartimentos isolados, cada qual apresentando as seguintes dimensões: $35 \mathrm{~cm}$ de altura, $39 \mathrm{~cm}$ de comprimento, $7 \mathrm{~cm}$ de largura no topo e $2 \mathrm{~cm}$ de largura no fundo.

O substrato utilizado para o cultivo das plantas foi uma mistura 1:1 em volume de areia (mesh 41) e "Sunshine mix \#1" (Sun Gro Horticulture Inc.), composto de 70 a $80 \%$ de esfagno, além de perlita, gesso, agente molhante e calcário dolomítico para ajuste do pH entre 6,0 e 6,5; utilizou-se uma quantidade invariável de substrato em todos os recipientes, de maneira a que o substrato tivesse a mesma massa específica, de $1,23 \mathrm{~g} \mathrm{~cm}^{-3}$.

Foram utilizados dois tratamentos, com diferentes concentrações de fósforo no substrato (alta e baixa, designadas por $+\mathrm{P}$ e $-\mathrm{P}$, respectivamente). $\mathrm{O}$ teor desejado de nutrientes em cada tratamento foi obtido pela adição de solução nutritiva ao substrato até que se atingisse um conteúdo volumétrico de água de $0,34 \mathrm{~cm}^{3} \mathrm{~cm}^{-3}$. A composição da solução nutritiva empregada foi a seguinte: $1,67 \mathrm{mmol} \mathrm{L}^{-1} \mathrm{KH}_{2} \mathrm{PO}_{4}, 5 \mathrm{mmol} \mathrm{L}^{-1} \mathrm{KNO}_{3}, 5 \mathrm{mmol} \mathrm{L}^{-1}$ $\mathrm{Ca}\left(\mathrm{NO}_{3}\right)_{2}$, e $2 \mathrm{mmol} \mathrm{L}^{-1} \mathrm{MgSO}_{4}$ para o tratamento $+\mathrm{P}$, e $0,33 \mathrm{mmol} \mathrm{L}^{-1} \mathrm{KH}_{2} \mathrm{PO}_{4}, 6,67 \mathrm{mmol} \mathrm{L}^{-1} \mathrm{KNO}_{3}, 5 \mathrm{mmol} \mathrm{L}^{-1}$ $\mathrm{Ca}\left(\mathrm{NO}_{3}\right)_{2}$, e $2 \mathrm{mmol} \mathrm{L}^{-1} \mathrm{MgSO}_{4}$ para o tratamento -P; em ambas as soluções, os micronutrientes foram adicionados nas seguintes concentrações: $0,5 \mathrm{mg} \mathrm{L}^{-1} \mathrm{~B}, 0,5 \mathrm{mg} \mathrm{L}^{-1} \mathrm{Mn}$, $0,05 \mathrm{mg} \mathrm{L}^{-1} \mathrm{Zn}, 0,02 \mathrm{mg} \mathrm{L}^{-1} \mathrm{Cu}$, e $0,01 \mathrm{mg} \mathrm{L}^{-1} \mathrm{Mo}$ (solução de Hoagland e Arnon, de acordo com Gauch, 1972). Embora a concentração de $P$ nas soluções nutritivas fosse de $51,6 \mathrm{mg} \mathrm{L}^{-1}$, e 10,3 $\mathrm{mg} \mathrm{L}^{-1}$ para os tratamentos $+\mathrm{P}$ e $P$, respectivamente, a concentração alcançada nos recipientes antes do cultivo e após adsorção pelo substrato sólido, foi de $15,5 \mathrm{mg} \mathrm{L}^{-1} \mathrm{P}(+\mathrm{P})$, e $8,5 \mathrm{mg} \mathrm{L}^{-1} \mathrm{P}(-\mathrm{P})$, medida com o extrator Bray.

Apenas uma semente foi depositada em cada compartimento dos recipientes, e se tal semente não germinasse em dois dias, aquela repetição era substituída. A superfície exposta do substrato nos recipientes foi coberta com papel alumínio após a emergência das plantas para evitar evaporação de água.

Logo após a semeadura, os recipientes foram colocados no interior de uma câmara de crescimento, com a temperatura do ar variando entre um valor mínimo diário de $19^{\circ} \mathrm{C}$ e um máximo diário de $27^{\circ} \mathrm{C}$, e com fotoperíodo constante de 15 horas durante todo o período experimental, de até 30 dias após a emergência das plantas.

Para a obtenção dos números e comprimentos médios dos trechos radiculares da raiz principal (ordem 0 , segundo a classificação de Rose, 1983) e das raízes laterais de ordens 1 a 3 (Rose, 1983), de acordo com sua posição de origem na raiz principal, adotou-se um processo 
de análise destrutivo, no qual as plantas foram removidas do solo a cada 5 dias $(5,10,15,20,25$ e 30 dias após emergência), sendo seu sistema radicular meticulosamente medido com uma régua flexível.

Os recipientes de acrílico possuíam paredes laterais deslizáveis, que permitiam a retirada de sistemas radiculares intactos em todas as amostragens.

Em cada amostragem, após a lavagem do sistema radicular, era determinado o comprimento da raiz principal pivotante (ordem 0 ), sendo em seguida realizado 0 secionamento do sistema radicular em trechos de $5 \mathrm{~cm}$ de comprimento ao longo da raiz principal. Eram então contados os números de raízes laterais de ordem 1 em cada trecho de $5 \mathrm{~cm}$ da raiz principal e em seguida 6 raízes laterais de ordem 1 eram retiradas de cada trecho, completamente ao acaso, e tinham seu comprimento determinado, com a finalidade de estimativa do comprimento médio de acordo com sua posição de origem na raiz principal. Em seguida eram escolhidos 6 trechos de $3 \mathrm{~cm}$ ao longo das raízes de ordem 1 identificadas de acordo com sua posição de origem, e era contado o número e medido o comprimento das raízes laterais de ordem 2, para estimativa do número médio de raízes de ordem 2 por centímetro de raiz de ordem 1, e seu comprimento médio. O mesmo procedimento era adotado para as raízes de ordem 3 , quando existentes.

Sendo assim, para cada tratamento, idade de amostragem e repetição foram obtidos o comprimento da raiz principal (ordem 0 ), e os respectivos números e comprimentos médios das raízes laterais das ordens 1, 2 e 3, de acordo com a posição de origem na raiz principal. Como o delineamento experimental (inteiramente casualizado, com parcelas subdivididas no tempo) contava com três repetições para cada idade de planta avaliada $(5,10,15,20,25$, e 30 dias após a germinação), calcularam-se índices topológicos para cada tratamento utilizando-se os valores médios.

\section{Índices Topológicos}

\section{Definições}

Em análises de sistemas radiculares utilizando-se conceitos de topologia, o sistema radicular é considerado como um conjunto de segmentos, e estes segmentos podem ser externos, quando se localizam entre um ponto de ramificação e um ponto de crescimento (meristema apical), ou internos (ou entrenós), quando se localizam entre dois pontos de ramificação. A esses segmentos ou a seu conjunto, vários índices podem ser definidos (Fitter, 1985; Fitter et al., 1991), os quais representam atributos funcionais do sistema radicular, em termos de aquisição de recursos do solo, ancoragem, ou outra função radicular qualquer.

No caso particular deste estudo, a função radicular estudada foi a aquisição de fósforo, recurso de mobilidade reduzida no solo. Os índices topológicos podem ajudar a identificar a configuração estrutural que representa o melhor desempenho na aquisição de fósforo pela planta, com o mínimo custo, isto é, a mínima quantidade de fotoassimilados direcionados para o sistema radicular.

Apresenta-se a seguir a definição dos principais índices topológicos, baseada nos conceitos de Fitter (1985, 1987).

Cada segmento radicular pode ser caracterizado por sua magnitude e caminho percorrido, e cada sistema radicular pode ser caracterizado por sua magnitude, caminho total percorrido e altitude.

A magnitude de um segmento $(m s)$ é dada pelo número de pontos de crescimento (meristemas apicais) se desenvolvendo a partir dele, portanto todos os segmentos externos têm magnitude 1 , e a magnitude de qualquer segmento interno é igual ao número de total de pontos de crescimento que se originam dele. A magnitude de um sistema radicular $(m)$ tem o mesmo valor de magnitude do segmento basal (aquele que se liga à parte aérea da planta), e é equivalente ao número total de meristemas apicais ou de segmentos externos do sistema radicular (Figura 1).

O caminho percorrido de um segmento (cs) é a soma do número de segmentos radiculares entre ele e o segmento basal (aquele que se conecta à parte aérea da planta), incluindo-se os extremos. O caminho total percorrido de um sistema radicular (ct) é a soma dos valores de caminhos percorridos de todos os segmentos externos, e é um número maior e diferente da soma do número de segmentos do sistema, pois alguns segmentos fazem parte de diversos caminhos percorridos (o segmento basal, por exemplo, faz parte dos caminhos percorridos de todos os outros segmentos).

A altitude de um sistema radicular (a) é o maior valor de caminho percorrido de um segmento no sistema, e representa o maior caminho, quanto ao número de segmentos, que um recurso do solo teria que percorrer de um segmento externo até a parte aérea da planta (Figura 2).

Sistemas radiculares topologicamente idênticos, isto é, com índices topológicos idênticos, podem ter aparências bastante distintas, se diferirem nos aspectos métricos de sua geometria (Fitter, 1987), tais como o comprimento dos segmentos radiculares. Estes aspectos métricos do sistema radicular são considerados em análises arquiteturais, mas são irrelevantes em análises topológicas.
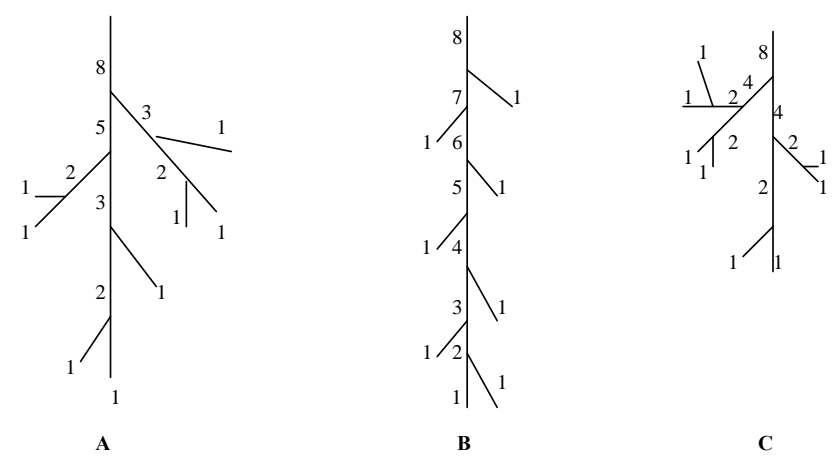

Figura 1 - Diagramas de sistemas radiculares mostrando valores de magnitude para cada segmento. Todos os diagramas representam sistemas radiculares de magnitude 8 , sen do B e C extremos topológicos (adaptado de Fitter, 1987). 

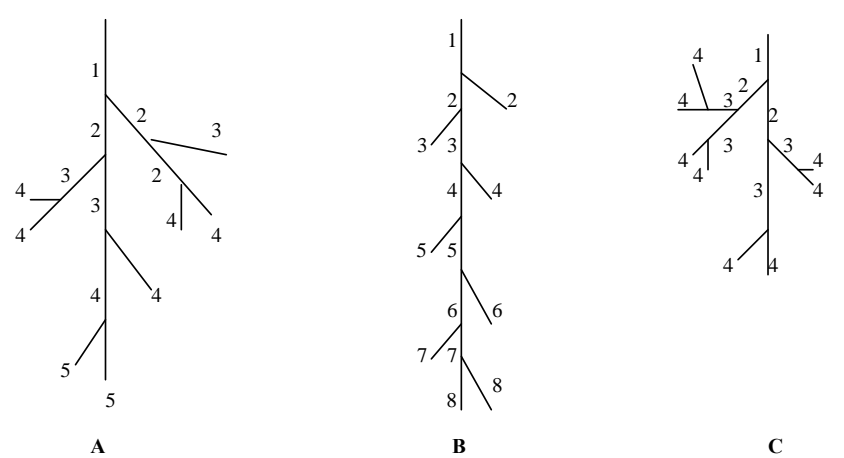

Figura 2 - Diagramas de sistemas radiculares mostrando os valores de caminho percorrido para cada segmento. A altitude (a) é o maior valor de caminho percorrido dentro do sistema radicular (5, 8 e 4, respectivamente) e o caminho total percorrido (ct) é a soma dos valores de caminhos percorridos de todos os segmentos radiculares externos (33, 43 e 32, respectivamente) (Adaptado de Fitter, 1987).

\section{Cálculo dos Índices Topológicos}

Com os dados obtidos experimentalmente, calcularam-se, para cada data de amostragem, valores dos seguintes índices topológicos, adotando-se os respectivos procedimentos:

(a) Magnitude: visto que a magnitude de um sistema radicular é equivalente ao número correspondente de meristemas apicais ou de segmentos radiculares externos, para efeito de cálculo seu valor em uma determinada data é o próprio número total de raízes, incluindo-se todas as ordens de desenvolvimento.

(b) Altitude: valores experimentais de altitude para cada idade de planta amostrada foram calculados a partir da soma dos números médios de raízes de todas as ordens, para cada profundidade de origem. A maior soma (profundidade com o maior número de segmentos radiculares) representa o caminho com o maior número de segmentos, ou seja, a altitude do sistema para aquela data.

Ajustaram-se curvas de variação da magnitude e da altitude do sistema radicular em função da idade da planta, mediante análises de regressão.

Para cada valor de magnitude do sistema radicular foram ainda calculados os valores máximos e mínimos de altitude correspondentes, segundo metodologia descrita em Werner \& Smart (1973) e Fitter (1985).

\section{RESULTADOS E DISCUSSÃO}

Verifica-se, na condição de menor teor de $\mathrm{P}$ no solo, uma magnitude pronunciadamente maior em relação à condição de maior fertilidade (Figura 3), refletindo maior número de pontos de crescimento. Trata-se de estratégia adaptativa adotada pela planta para melhor explorar o solo em busca do nutriente escasso, altamente imóvel no solo, no caso o fósforo. Para nutrientes altamente móveis no solo, como o nitrato, não seria esperada a mesma alteração estrutural do sistema radicular, pois raízes em grande número acabariam competindo entre si pelo mesmo recurso (Harper et al., 1991).

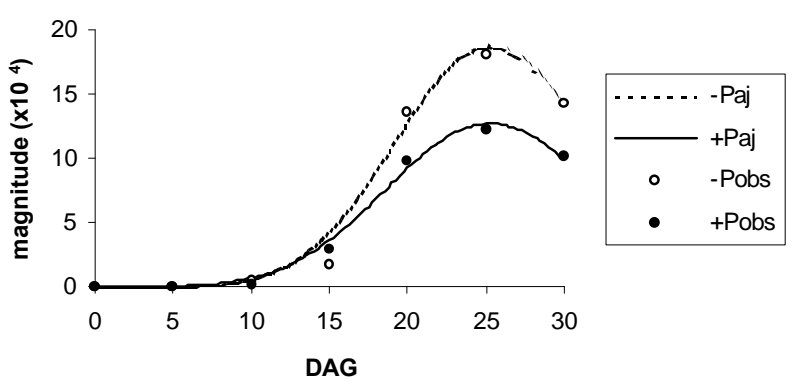

Figura 3 - Curvas ajustadas da variação temporal da magnitude do sistema radicular para ambos os tratamentos (-Paj e +Paj, baixo e alto teor de $P$, respectivamente). Pontos (-Pobs $e$ +Pobs, 8,5 e 15,5 $\mathrm{mg} \mathrm{L}^{-1} \mathrm{P}$, respectivamente) representam valores calculados a partir dos dados experimentais. DAG = dias após a germinação.

O que explica essa hipótese é o diâmetro da zona de depleção do nutriente escasso que se forma ao redor de cada segmento do sistema radicular que o está absorvendo. Nutrientes com baixa difusividade no solo, como o fosfato, apresentam zonas de depleção com diâmetro bastante reduzido, portanto a produção de um grande número de raízes proporcionaria melhor exploração do solo em busca de tal recurso, já que elas não competiriam entre si. Por outro lado, no caso dos nutrientes com alta difusividade no solo, desenvolvem-se zonas de depleção com maiores diâmetros ao redor dos segmentos radiculares que os estão absorvendo, e se as zonas de depleção de dois segmentos radiculares se encontrassem (overlap), a eficiência de aquisição do nutriente (massa de nutriente absorvido por massa de tecido radicular produzido) seria reduzida. Robinson (1991) apresenta uma interessante abordagem sobre zonas de depleção.

Quanto à altitude do sistema radicular, seu valor máximo é sempre associado à uma configuração do tipo "espinha-de-peixe", que otimiza uma exploração acelerada do solo em busca do recurso escasso (maior volume de solo efetivamente ocupado por raízes), e seu valor mínimo associado a uma configuração dicótoma, a qual proporciona alta eficiência de aquisição do recurso (quantidade de recurso absorvido por quantidade de raiz) em um volume restrito de solo, no caso de recursos com baixa difusividade no solo (Fitter et al., 1991). Sistemas radiculares reais apresentam configuração intermediária entre esses dois extremos, visto que a melhor estratégia adaptativa da planta representa equilíbrio entre eficiência de exploração do solo e eficiência de aquisição do recurso do solo, tendendo mais para um dos lados dependendo do genótipo e das condições edáficas (Tiffney \& Niklas, 1985).

No tratamento com menor teor de fósforo, houve exploração mais acelerada do solo, representando estratégia eficiente na busca pelo recurso escasso, conforme refletida pelos maiores valores de altitude (Figura 4a). No entanto, quando se comparam os valores medidos 
A

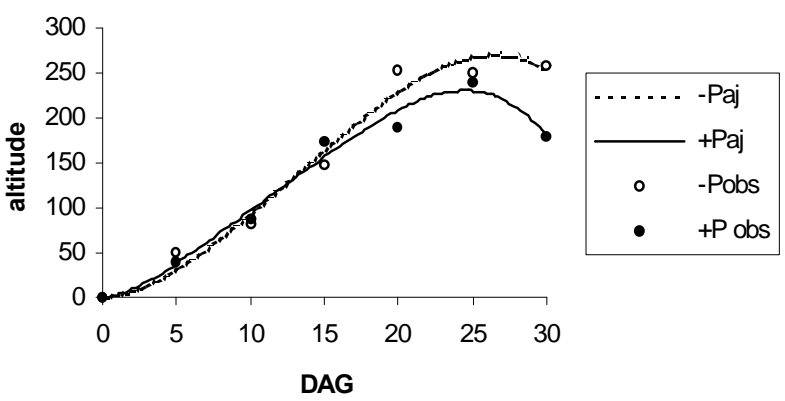

B

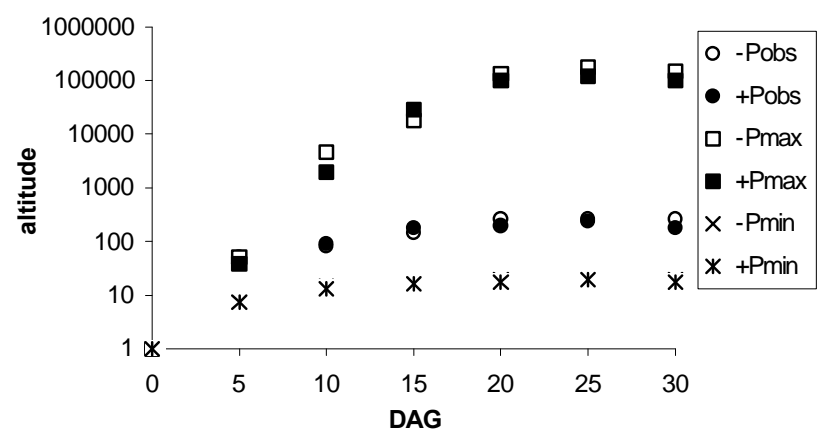

Figura 4 - (A) Curvas ajustadas da variação temporal da altitude do sistema radicular para ambos os tratamentos $(-P a j e+P a j$, baixo e alto teor de $P$, respectivamente). Pontos (-Pobs $e$ +Pobs, 8,5 e $15,5 \mathrm{mg} \mathrm{L}^{-1} \mathrm{P}$, respectivamente) representam valores calculados a partir dos dados experimentais. (B) Valores estimados máximos (-Pmax e +Pmax) e mínimos (-Pmin e -Pmax) de altitude (Werner \& Smart, 1973), plotados juntamente com os valores experimentais de al titude (-Pobs e +Pobs), para ambos os tratamentos. DAG = dias após a germinação.

de a com os valores máximos e mínimos calculados (Figura 4b), verifica-se que em ambos os tratamentos a estratégia da planta é mais voltada para eficiência de aquisição (os valores medidos são mais próximos dos valores mínimos que dos máximos), possivelmente uma característica da espécie ou do genótipo (Fitter, 1985, 1987).

Outro fator interessante é que se o sistema radicular da planta tendesse muito para a configuração "espinha-de-peixe", este seria muito suscetível a danos físicos, pois cada segmento radicular interno seria ponto de passagem de água e nutrientes provenientes de um grande número de outros segmentos. Em concordância, verifica-se que a arquitetura radicular desvia-se da forma "espinha de peixe" em ambos os tratamentos (Fitter, 1985), conforme indicado pelo desvio dos valores medidos em relação ao valores máximos de altitude (Figura 5).

Valores de magnitude e altitude podem ser calculados não apenas para o sistema radicular inteiro, mas também para pequenos trechos do mesmo. Em estudos deste tipo poder-se-ia verificar o comportamento de sistemas radiculares em solos heterogêneos, com bolsões de maior ou menor fertilidade em relação à média

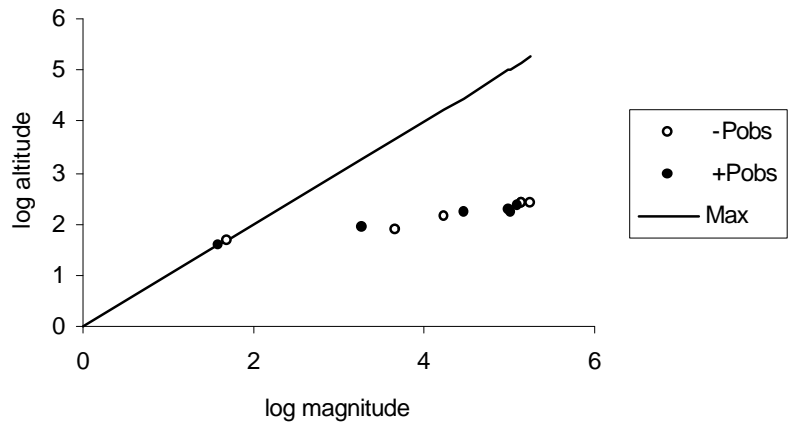

Figura 5 - Altitude do sistema radicular em função da magnitude, mostrando o desvio dos valores experimentais observados (-Pobs e +Pobs, representando os tratamentos com 8,5 e $15,5 \mathrm{mg} \mathrm{L}^{-1} \mathrm{P}$, respectivamente) em relação aos valores máximos ( $\max$ ) estimados segundo metodologia descrita em Werner \& Smart (1973).

do perfil do solo. Trechos do sistema radicular que se desenvolvem em bolsões de maior fertilidade apresentariam valores de altitude relativamente menores, e o uso de índices topológicos permitiria o estudo da variabilidade e compensação existentes em sistemas radiculares sob estas condições (Robinson, 1996).

\section{CONCLUSÕES}

- Índices topológicos tais como magnitude e altitude podem ser utilizados para identificar alterações estruturais no sistema radicular, que refletem adaptações ao ambiente edáfico.

- Plantas que crescem em solos pobres em fósforo apresentam valores de magnitude e altitude de seus sistemas radiculares comparativamente maiores em relação a plantas crescendo em solos férteis, indicando estratégia adaptativa da planta em direção à maior eficiência de exploração do solo.

\section{AGRADECIMENTOS}

À Fundação de Amparo à Pesquisa do Estado de São Paulo (FAPESP) e ao United States Department of Agriculture (USDA), pelo apoio financeiro concedido, e ao Dr. T. C. Hsiao, da University of California, Davis, EUA, pela colaboração no delineamento experimental.

\section{REFERÊNCIAS BIBLIOGRÁFICAS}

FITTER, A.H. Functional significance of root morphology and root system architecture. In: FITTER, A.H. (Ed.) Ecological interactions in soil. Oxford: Blackwell, 1985. p.87-106

FITTER, A.H. An architectural approach to the comparative ecology of plant root systems. New Phytologist, v.106, p.61-77, 1987. Suplement.

FITTER, A.H.; STICKLAND, T.R.; HATVEY, M.L.; WILSON, G.W. Architectural analysis of plant root systems: 1. Architectural correlates of exploitation efficiency. New Phytologist, v.118, p.375-382, 1991.

GAUCH, H.G. Inorganic plant nutrition. Stroudsburg: Dowden, Hutchinson \& Ross, 1972. 488p. 
HARPER, J.L.; JONES, M.; SACKVILLE-HAMILTON, N.R. The evolution of roots and the problems of analysing their behaviour. In: ATKINSON, D. (Ed.) Plant root growth: an ecological perspective. Oxford: Blackwell, 1991. p.3-22.

LYNCH, J. Root architecture and plant productivity. Plant Physiology, v.109, p.7-13, 1995.

LYNCH, J.P.; BEEBE, S.E. Adaptation of beans (Phaseolus vulgaris L.) to low phosphorus availability. HortScience, v.30, p.1165-1171, 1995.

LYNCH, J.P.; NILSEN, K.L.; DAVIS, R.D.; JABLOKOW, A.G. SimRoot: modelling and visualization of root systems. Plant and Soil, v.188, p.139-151, 1997.

McCULLY, M. How do real roots work? Some new views of root structure. Plant Physiology, v.109, p.1-6, 1995.

ROBINSON, D. Roots and resource fluxes in plants and communities. In: ATKINSON, D. (Ed.) Plant root growth: an ecological perspective. Oxford: Blackwell, 1991. p.103-130.
ROBINSON, D. Variation, co-ordination and compensation in root systems in relation to soil variability. Plant and Soil, v.187, p.57-66, 1996.

ROSE, D. A. The description of the growth of root systems. Plant and Soil, v.75, p.405-415, 1983.

TIFFNEY, B.H.; NIKLAS, K.J. Clonal growth in land plants: a palaeobotanical perspective. In: JACKSON, J.B.C.; BUSS, L.W.; COOK, R.E. (Ed.) Population biology and evolution of clonal organisms. New Haven: Yale University Press, 1985. p.35-66.

WERNER, C.; SMART, J.S. Some new methods of topologic classification of channel networks. Geographical analysis, v.5, p.271-295, 1973.

Recebido em 20.09.99 